\title{
Interdisziplinäre Betreuung von Schwangeren mit Drogenabhängigkeit und ihren Neugeborenen
}

\author{
Andreas ]. Gerhardt, Carmen Aschka
}

\author{
Drogen abhängige werdende Mütter zu betreuen, ist eine interdisziplinäre Herausforderung \\ von der Schwangerschaft über die Geburt bis zum Wochenbett. Wie begleiten wir diese \\ Frauen und ihre Familie angemessen, wertschätzend und zugewandt durch diese schwierige \\ Lebensphase? Wie bewältigen wir es, das Leid zu erleben, das der Drogenmissbrauch her- \\ vorruft? Welche Chancen können wir bieten? Das Autorenteam beantwortet diese Fragen \\ aus Sicht der klinischen Praxis und auf Basis von Fachliteratur und Leitlinien.
}

\section{Fallbeispiel: Ein unsanfter Einstieg}

Harzklinikum, Wernigerode. Eigentlich sollte die Frühgeborenenstation unseres Perinatalzentrums ein ruhiger, stiller Ort sein. Hier bieten wir für Babys, die Wochen vor ihrem Geburtstermin das Licht der Welt erblicken, und für deren Mütter eine Versorgung in reizarmer Umgebung. Doch heute dringen schrille Schreie aus einem der Bettchen. Eine Schwester bemüht sich, das Kind zu umgrenzen, zu halten, etwas Glukoselösung zum Saugen anzubieten, dann das sich erbrechende Baby wieder hoch zu nehmen. Es wimmert, überstreckt sich opistoton, das Gesichtchen schmerzverzerrt. An Kopf und Körper fallen Hämatome und Ödeme auf. Jede Berührung der Schwester löst heftige Schmerzreaktionen aus. Die Windel ist bei jeder Versorgung voll von wässrigem Durchfall. Der Po wird wund und ist bald eine offene nässende Wunde. Erst als es etwas Opiat-Lösung bekommt, beruhigt es sich und fällt in einen unruhigen Schlaf, schreckt jedoch bei der kleinsten Störung hoch.

Es ist der zweite Lebenstag eines Neugeborenen, dessen Mutter mit Polamidon substituiert wird. Sie beteuerte uns, auf der niedrigsten Substitutionsdosis ihres Lebens zu sein.

Egal, wie gut organisiert und geplant man die Frau durch die Schwangerschaft begleitet: Den Entzug, den die Mutter nicht schafft, muss das Neugeborene durchleben. Darauf muss sich das interdisziplinäre Team fachlich vorbereiten und in der Praxis Hand in Hand zusammenarbeiten.

\section{GLOSSAR}

\section{Drogen und Sucht}

Nach der Definition der Weltgesundheitsorganisation (WHO) gilt als Droge jede Substanz, die in einem lebenden Organismus Funktionen zu verändern vermag. Hierzu zählen nicht nur illegale Drogen, sondern auch sog. Genussmittel wie Alkohol, Tabak und Kaffee sowie Schmerz- und Beruhigungsmittel.

Drogen lassen sich grundsätzlich nach ihrer vorherrschenden Wirkung einteilen:

- stimulierend wirken z. B. Methamphetamin, Kokain oder Koffein

- dämpfend und angstlösend wirken z.B. Alkohol, Cannabis, Opioide oder Benzodiazepine

- außerdem gibt es die Gruppe der halluzinogenen Substanzen wie beispielsweise LSD oder Psylocibin

Es kann in der Wirkung dosisabhängig Überschneidungen geben. Drogen können je nach Substanz also ganz unterschiedlich wirken. Entsprechend unterschiedlich stellt sich auch der Entzug von der jeweiligen Droge dar. Die Risiken fürs Kind potenzieren sich bei multiplem Substanzgebrauch der Mutter, da sich die Wirkungen der einzelnen Suchtmittel gegenseitig beeinflussen und verstärken können. Eine Sucht ist eine chronische Krankheit, die zu anhaltend fortbestehenden Hirnfunktionsänderungen führt. Dabei wird Sucht als das körperliche und geistige Verlangen nach einer Substanz definiert, 
nach deren Konsum sich das physische und psychische Wohlbefinden der süchtigen Person massiv verbessert. Trotz des eigenen Wissens um ihre Abhängigkeit von dieser Substanz gelingt es nur wenigen Konsumenten, die Sucht zu überwinden. Zu Rückfällen kommt es nicht aus freiem Willen.

\section{Anamnese von Drogenkonsum in der Schwangerschaft}

Es gibt keine verlässliche Statistik bezüglich Drogenkonsums in der Schwangerschaft in Deutschland, wohl aber zur Häufigkeit von Drogenkonsum generell, auch nach Altersgruppen unterteilt. Die einzigen Drogen, zu denen es Daten bezüglich der Häufigkeit des Konsums in der Schwangerschaft gibt, sind Alkohol und Nikotin. Alkohol ist zugleich die außer- und innerhalb der Schwangerschaft mit Abstand am häufigsten konsumierte und am meisten unterschätzte Droge. Außerdem spielt Alkohol eine wichtige Rolle im Sinne eines Beikonsums der Konsumentinnen illegaler Drogen. Gleiches gilt auch für Nikotin. Illegale Drogen werden häufiger von jüngeren Menschen konsumiert und so sind insbesondere Frauen im gebärfähigen Alter betroffen. [5][6][7]

\section{Alkohol}

In der Schwangerschaft geben je nach Untersuchung in Deutschland 14-58\% der Schwangeren an, Alkohol zumindest gelegentlich zu konsumieren. Hierbei ist der Alkoholkonsum vor Bekanntwerden der Schwangerschaft bereits ausgeschlossen. Ein exzessiver Alkoholkonsum im Sinne eines Rauschtrinkens wird von 1,2-3,5\% der Schwangeren betrieben, wiederum nach Bekanntwerden der Schwangerschaft. Eine kleinere Studie zeigte Prävalenzen für das Rauschtrinken $1 x /$ Monat von $4 \%$ und weitere $4 \%$ der Schwangeren gaben an, Alkohol mehrmals pro Woche zu konsumieren.

Schädigungen, die durch intrauterine Alkoholexposition hervorgerufen sind, werden unter dem Oberbegriff Fetale Alkoholspektrumstörung (FASD - fetal alcohol spectrum

disorder) zusammengefasst [20]. Die fetale Alkoholspektrumstörung tritt in Deutschland bei etwa 10000 Kindern pro Jahr auf. Hierbei ist von einer hohen Dunkelziffer auszugehen. Kindliche Schädigungen durch Alkohol liegen bei mindestens $1 \%$ und damit deutlich höher als das generelle Risiko für chromosomale Schädigungen inklusive Trisomie 21 (0,2\%) und auch Schädigungen durch prä- und/oder intrapartale Asphyxie mit Cerebralparese $(0,2-0,3 \%)$.

Diese Daten stehen in einem krassen Gegensatz zu unseren im Harzklinikum Wernigerode anamnestisch erhobenen Fällen von Alkoholkonsum in der Schwangerschaft. Kaum eine Frau gibt an, Alkohol zu trinken, auch nicht auf direkte Nachfrage. Deshalb ist es wichtig zu wissen, welche Risikofaktoren es für Alkoholkonsum in der Schwangerschaft gibt.

\section{INFO \\ Risikofaktoren für Alkoholkonsum in der Schwangerschaft}

Folgende Risikofaktoren stehen in Zusammenhang mit Alkoholkonsum in der Schwangerschaft [1][4]:

- Höheres maternales Alter (>25 Jahre bzw. >30 Jahre)

- kein Migrationshintergrund

- hoher sozioökonomischer Status (höhere Bildung, höheres Einkommen etc.)

- hoher Alkoholkonsum vor der Schwangerschaft

- Konsum illegaler Drogen und/oder Nikotinkonsum

- ungewollte Schwangerschaft; Z. n. Abruptio

- alleinstehend

- psychische Störungen

Eine ausführliche Beschreibung aller Risikofaktoren finden Sie im Artikel „Fetale Alkoholspektrumstörung - Diagnose und frühe Förderung“ von Dr. Mirjam N. Landgraf, der in DIE HEBAMME 5/2017 erschienen ist [20].

\section{Merke}

Alkoholkonsum in der Schwangerschaft erschließt sich nicht auf den ersten Blick und wird selten im Anamnesegespräch angegeben. Für die frühe Prävention ist es daher wichtig, dass betreuende Fachkräfte Risikofaktoren abklären, die Frauen, ihre Partner und die anderen Kinder beobachten und sensibel nach dem Alkoholkonsumverhalten in der Familie fragen.

\section{Nikotin}

Etwas anders als beim Alkoholkonsum sieht die Situation beim Rauchen aus. In aller Regel wird ein Nikotinkonsum bereitwillig angegeben, wenn auch die Menge der täglich konsumierten Zigaretten zumeist deutlich höher liegt als dokumentiert. In Deutschland rauchen etwa $10 \%$ der Frauen trotz bestehender Schwangerschaft weiter. Dies hat, durch die wohlbekannten Gefahren für das Kind, insbesondere Plazentainsuffizienz und deren Folgen, auch Konsequenzen für das geburtshilfliche Vorgehen in Terminnähe. So soll laut Leitlinie der Deutschen Gesellschaft für Gynäkologie und Geburtshilfe der Risikofaktor Nikotinabusus für den IUFT unbedingt in die Entscheidung über die Geburtsplanung mit einfließen und ggf. eine Einleitung gegenüber abwartendem Verhalten bei Terminüberschreitung bevorzugt werden [2][6]. 


\section{Cannabis}

Cannabis erfährt gegenwärtig eine zunehmende gesellschaftliche Akzeptanz. Der Konsum von Cannabis ist anamnestisch meist unproblematisch zu erheben. Lange Zeit galt er als unproblematisch bezüglich kindlicher Schäden. Durch Studien in Ländern, in denen der Konsum legal ist, z.B. in einigen Bundesstaaten der USA oder in den Niederlanden, wissen wir heute mehr über die Folgen: Cannabis wirkt neurotoxisch und wird mit Anenzephalie und IUFT in Verbindung gebracht. Aus diesen Ländern wissen wir auch, dass Cannabis mit einer Häufigkeit von 2-5\% in der Schwangerschaft konsumiert wird. [9][10][11]

\section{Methamphetamine und Heroin}

Frauen, die die wesentlich selteneren „harten“ Drogen wie Methamphetamine (z. B. Crystal) oder Heroin konsumieren, fallen spätestens in Entzugssituationen auf. Manchmal wird der Konsum aber auch vorher angegeben, dies gilt insbesondere, wenn sich die Schwangere in einem Substitutionsprogramm befindet (Polamidon, Subutex). Es ist wichtig, zu wissen, dass ein moderater Konsum illegaler, auch harter Drogen nicht im äußeren Erscheinungsbild auffallen muss. Die häufig in Umlauf gewesenen „Vorher-Nachher-Bilder“ von Crystalkonsumenten kommen in der Regel aus Ländern, in denen das Gesundheitssystem nicht vergleichbar mit dem unseren ist und sind somit nicht auf Deutschland übertragbar. Menschen, die stimulierende Drogen konsumieren, können durchaus ganz normal gesellschaftlich eingebunden sein und einem geregelten Beruf nachgehen.

INFO

Auswirkungen des Chrystalkonsums auf Schwangerschaft und Geburt

Die höchste Prävalenz für den Konsum von Chrystal

bei Frauen liegt in der Altersspanne zwischen 20 und 30 Jahren. Für die Schwangerschaft und Geburt ergeben sich zahlreiche Probleme durch Crystalkonsum: Durch sexuelle Enthemmung kommt es vergleichsweise häufig zu Geschlechtsverkehr ohne Kontrazeption und zu wechselnden Sexualpartnern mit dem Risiko, sich mit sexuell übertragbaren Erkrankungen zu infizieren, z. B. HIV/Aids. Durch einen gestörten Menstruationszyklus der Konsumentinnen bestehen häufig eine Terminunklarheit sowie eine späte Schwangerschaftserfassung. Etwa $20 \%$ werden nach der 20 . SSW erstmalig zur Schwangerschaftsvorsorge vorstellig, bei $14 \%$ gibt es keinerlei Vorsorge. Das Risiko für Frühgeburtlichkeit sowie fetale Retardierung ist um den Faktor 3 erhöht, außerdem steigt das Risiko für eine vorzeitige Plazentalösung durch die vasokonstruktive Wirkung von Crystal über eine Adrenalinausschüttung. Aus diesem Grund ist auch eine pränatale Asphyxie bei mangelhafter Plazentaperfusion möglich. Diese Problematik besteht bei allen stimulierenden Drogen (z. B. Kokain, Ecstasy). Des Weiteren kann es durch die neurotoxische Wirkung von Crystal zu Organschäden wie Mikrozephalie kommen. Außerdem werden vermehrt kardiale Schädigungen und Nierenschäden sowie Lippenkiefergaumenspalten beobachtet. Während der Geburt ist bei Frauen, die Chrystal konsumieren, mit einer verminderten maternalen Belastbarkeit bei gesteigerter Aggressivität und geringer Frustrationstoleranz zu rechnen. [3][8]

\section{Schwangerschaftsvorsorge bei Drogenkonsum}

Wesentlich für die Vorsorge ist, konsequent jede Schwangere nach Alkohol-, Nikotin- und Drogenkonsum zu fragen und dies zu dokumentieren. Keinesfalls sollte ein Drogenscreening ohne Zustimmung der Schwangeren erfolgen, weil das notwendige Vertrauensverhältnis dadurch nachhaltig gestört werden kann.

Bei bekanntem Drogenkonsum sollten eine Organsonographie sowie regelmäßige Wachstums- und Dopplerkontrollen durchgeführt werden.

Eine präpartale Vorstellung in der Geburtsklinik ist sinnvoll, da gerade in der Ausnahmesituation während der Geburt eine bekannte Umgebung und bekannte Gesichter das notwendige Vertrauen schaffen können. Außerdem kann so frühzeitig Kontakt zu den Kinderärztinnen und Kinderärzten aufgenommen werden, die sich auf das Kind einstellen und der Schwangeren einen möglichen Ablauf für das Kind nach der Geburt erklären können.

Im Rahmen der Vorsorge können und sollten der Frau auch Hilfen angeboten werden (s.u. „Runder Tisch“). Der Kontakt zu einer Familienhebamme kann bereits hergestellt werden, möglicherweise wird auch Hilfe bei Behördengängen benötigt. Mit Einverständnis der Schwangeren ist auch die Weiterleitung an eine Suchtberatungsstelle oder an das Jugendamt denkbar. Diese Angebote sollten ohne Druck erfolgen, damit sich die Frau nicht aus Angst, man könne ihr das Kind wegnehmen, der Vorsorge entzieht.

\footnotetext{
Merke

Bei Schwangeren mit Drogenkonsum sollten eine Organsonographe, regelmäßige Wachstums- und Dopplerkontrollen durchgeführt werden. Sinnvoll ist die vorgeburtliche Vorstellung in der Geburtsklinik und die frühe Vernetzung mit einer Familienhebamme und Suchtberatungsstelle.
} 


\section{Betreuung drogenabhängiger Schwangerer während der Geburt}

Wir wissen, dass der Umgang mit drogenabhängigen Schwangeren schwierig sein kann. Während der Geburt potenzieren sich diese Schwierigkeiten oftmals. Hebamme und Geburtshelfer/in sollten sich bewusstmachen, dass es sich bei einer Sucht um eine Erkrankung handelt, die häufig mit einer anhaltenden Hirnfunktionsänderung einhergeht. Das aktuelle Verhalten stellt also keinen persönlichen Angriff dar, auch wenn es so aussehen mag. Insbesondere gilt zu beachten:

- Keiner drogenabhängigen Gebärenden sollten Schmerzmittel vorenthalten werden. Dies geschieht häufig aus Angst, eine Abhängigkeit zu unterhalten.

- Je nachdem, welche Drogen konsumiert werden, muss die Schmerztherapie während der Geburt angepasst werden, oft sind hohe Dosen notwendig. Bei Frauen in Substitutionsprogrammen kann eine entsprechende Erhöhung des Substitutionsstoffes Sinn machen, andere Opioide können problematisch sein.

- Eine PDA als regionales Verfahren ist in der Regel unproblematisch bei Drogenkonsum.

Falls eine präpartale Vorstellung in der Geburtsklinik erfolgt, kann hier in Absprache mit den Schmerztherapeuten ein konkretes Vorgehen bereits festgelegt werden.

\section{Interdisziplinäres Management nach der Geburt}

Im Folgenden geben wir Einblicke in die interdisziplinäre Betreuung von Neugeborenen und deren Familien am Harzklinikum Wernigerode nach einer durch Substanzabusus geprägten Schwangerschaft.

\section{Anamnese des Neugeborenen}

In manchen Fällen wird der Drogenmissbrauch der Mutter während der Schwangerschaft erst nach der Geburt festgestellt. Die wenigsten Neugeborenen werden jedoch wie im eingangs beschriebenen Fall auffällig. Oft zeigen sie vielmehr unspezifische Zeichen, z.B. Unruhe, heftiges Saugbedürfnis und Berührungsempfindlichkeit. Dies sind Zeichen, die bei anderen Erkrankungsbildern (wie Infektion, Dehydratation, Kernikterus oder leichtem Hirnödem nach Asphyxie) ebenfalls auftreten können. Daher kommt der Anamnese des Neugeborenen und einem gewissen Gespür der Untersuchenden für besondere Verhaltensauffälligkeiten ein hoher Stellenwert in der Detektion von Substanzmissbrauch zu. Meist ergibt sich aus verschiedenen Puzzle-Teilen der Verdacht auf einen intrapartalen Drogenabusus. Und erst in der erbetenen Urinprobe der Mutter oder des Neugeborenen findet sich der Beweis dafür. Damit beginnt die Suchtberatung im Wochenbett und auf unserer Neonatologie.
Klarer ist der Fall, wenn der Drogenabusus schon in der Schwangerschaft bekannt wurde, die Mutter dahingehend eine Therapie und Betreuung erfahren hat. Hier kommt dem „Runden-Tisch“ eine besondere Rolle zu: Dieses Treffen findet idealerweise bereits im 2. Trimenon statt und hat das Ziel, alle Beteiligten zusammenzubringen und einen gemeinsamen Wissensstand zu erarbeiten. Teilnehmer sind die Schwangere selbst, ihr Suchttherapeut/Substitutionsmediziner, ein Vertreter des Jugendamts, ein ambulanter Gynäkologe und Perinatalmediziner. Diese Abstimmung schafft Transparenz und Effizienz in der Organisation und Planung des weiteren Lebens der wachsenden Familie.

\section{Das Neonatale Abstinenzsyndrom (NAS)}

Je nach Substanz treten bei den Neugeborenen unterschiedliche Symptome zutage. Nicht jedes Neugeborene zeigt nach Schwangerschaft mit Drogenabusus das Vollbild der Symptome eines Neonatalen Abstinenzsyndroms wie muskuläre Hypertonie, Hyperexzitabilität, Spucken, Erbrechen, Diarrhö oder/und Krämpfe [16][17].

Grob verallgemeinert kann man sagen: Bei den Substanzen kehren sich die Wirkungen um und in der durch die Geburt erzwungenen Abstinenz reagieren die Säuglinge entsprechend advers. Aufputschende Mittel ziehen nach einer Phase der Irritation häufig Lethargie und Trinkschwäche nach sich. Sedierende, schmerzlindernde Mittel führen dagegen zu Vulnerabilität, gesteigertem Schmerzempfinden und vermehrtem Saugbedürfnis.

\section{Neonatales Abstinenzsyndrom nach Opiatabusus}

Am deutlichsten zeigt sich das Neonatale Abstinenzsyndrom im Falle eines Opiatabusus oder einer Substitution mit Methadon. Während die Droge schmerzstillend, sedierend, angstlösend wirkt und den Muskeltonus senkt (Harnverhalt, Verstopfung), so sind nach der Geburt Schmerzen, Hyperexzitabilität, exzessives Saugen, Spucken, Erbrechen und Durchfälle zu beobachten. Die auf unseren Nervenzellen natürlicherweise vorkommenden Opioidrezeptoren benötigen wir zur interzellularen Kommunikation für eine normale Vernetzung insbesondere im Nervensystem. Wenn ein heranwachsender Fötus unter stetiger Sedierung mit Opiaten diese Vernetzung seiner Nervenzellen erreichen will, so muss er das 1000fache dieser Rezeptoren auf den Zelloberflächen ausbilden, damit noch einige „nichtblockierte“ eine normale Funktion aufbauen können.

Nach der Geburt stoppt der ständige Zustrom von Opiat über die Nabelschnur. Die hochregulierten Opiat-/ Schmerzrezeptoren sind nun frei und senden unblockiert kleinste Irritationen als 1000-fach verstärktes Schmerzsignal an das Neugeborenengehirn. Untersuchungen haben ergeben, dass diese Vulnerabilität zeitlebens zumindest teilweise bestehen bleibt. Verschiedene 
Forscher sehen hier auch eine der bedeutendsten Ursachen für die hohe Affinität dieser Kinder, später ebenfalls eine Sucht auszubilden.

Therapeutisches Ziel ist das konsequente Herunterregulieren dieser Rezeptoren, indem man sie mit einer nach und nach immer niedrigeren Dosis von Opioiden blockiert. Abruptes Absetzen kann zu einem Rebound des NAS bis hin zu epileptischen Krämpfen führen.

Bei Polamidonsubstitution ist zudem eine Thrombozytenfunktionsstörung zu beobachten, die mit vermehrten Hämatomen und Petechien bei Spontangeborenen einhergeht.

\section{Entzugssymptomatik bei Nikotinkonsum}

Das weitverbreitete Rauchen wird seitens seiner Entzugssymptomatik meist unterschätzt. Einige Experten vergleichen die Stärke der Abhängigkeit mit der von Heroin. Die Dauer der Entzugssymptomatik beim Neugeborenen ist jedoch deutlich kürzer, wenn auch die Symptome den Kindern von opiatabhängigen Müttern ähneln.

Hyperexzitabilität, exzessives Saugen, Spucken, Erbrechen und Durchfälle sistieren meist 3-4 Tage nach
Geburt und sind mit nichtmedikamentösen Methoden recht gut zu beherrschen. Nur selten müssen sedierende Substanzen, wie Phenobarbital verabreicht werden.

Wenn die ersten Tage überstanden sind, entwickeln sich die Kleinen meist ganz normal. Babys von Rauchern begleitet aber ein Paket von erhöhten Risiken, wie z.B. SID (plötzlicher Kindstod), Allergien, Asthma, Neurodermitis, hyperreagibles Bronchialsystem.

\section{Entzugssymptomatik bei Abhängigkeit von Stimulantien}

Amphetamine, Methamphetamin, Kokain etc. sind Stimulantien, bei denen die Entzugserscheinungen eher durch einen niedrigen Muskeltonus und Trinkschwäche geprägt sind. Sie können aber auch ein buntes Bild aus Unruhe, vermehrtem Saugbedürfnis, Tremor und Irritabilität zeigen, die ihr Maximum um den 3. Lebenstag haben [16].

\section{Entzugssymptomatik bei Cannabiskonsum}

Die Neugeborenen von Cannabis konsumierenden Müttern zeigen in den ersten Lebenstagen häufig wenig Symptome, die klar dem Drogenabusus zuzuordnen wären. Durch die Legalisierung von Cannabis häufen sich

-Tab. 1 Drogen und ihre Entzugssymptomatik beim Neugeborenen.

\begin{tabular}{|c|c|c|c|}
\hline Droge & Symptome & Beginn & Dauer \\
\hline Alkohol & $\begin{array}{l}\text { Hyperaktivität, Schreien, Irritabilität, Saugschwä- } \\
\text { che, vermehrtes Saugbedürfnis, Tremor, Krämpfe, } \\
\text { gestörter Schlaf, Schwitzen }\end{array}$ & 3-12 Stunden & 18 Monate \\
\hline Barbiturate & $\begin{array}{l}\text { Irritabilität, heftige Krämpfe, Hyperakusis, schril- } \\
\text { les Schreien, vasomotorische Instabilität, Diarrhö, } \\
\text { Unruhe, hoher Muskeltonus, vermehrtes Saugbe- } \\
\text { dürfnis, Erbrechen, unruhiger Schlaf }\end{array}$ & 1-14 Tage & 4-6 Monate \\
\hline Coffein & Nervosität, Erbrechen, Bradykardie, Tachypnoe & ab Geburt & 1-7 Tage \\
\hline $\begin{array}{l}\text { Langwirksame Benzodiazepine } \\
\text { (Chlordiazepoxide) }\end{array}$ & Irritabilität, Tremor & Tage - 3 Wochen & 9 Monate \\
\hline $\begin{array}{l}\text { Kurzwirksame Benzodiazepine } \\
\text { (Diazepam) }\end{array}$ & $\begin{array}{l}\text { muskuläre Hypotonie, Trinkschwäche, Hypother- } \\
\text { mie, Apnoen, arterielle Hypertonie, Tachypnoe, } \\
\text { Hyperreflexie, Hyperexzitabilität, Erbrechen }\end{array}$ & Stunden - Wochen & 8 Monate \\
\hline Trizyklische Antidepressiva & Hypothermie, Zyanose, Zittrigkeit, & Stunden - Wochen & 4 Tage \\
\hline Ethchlorvynol (GABAerges Hypnotikum) & $\begin{array}{l}\text { Lethargie, Erbrechen, Irritabilität, vermehrtes Sau- } \\
\text { gen, Saugschwäche, muskuläre Hypotonie }\end{array}$ & & 10 Tage \\
\hline Hydroxyzin (Antihistaminikum) & $\begin{array}{l}\text { Tremor, Lethargie, Erbrechen, Irritabilität, schrilles } \\
\text { Schreien, Myoklonien, Tachypnoe, Tachykardie, } \\
\text { vermehrtes Saugen, Saugschwäche }\end{array}$ & & 5 Wochen \\
\hline Urethane (Meprobamat) & $\begin{array}{l}\text { Irritabilität, heftige Krämpfe, Bauchschmerzen, } \\
\text { gestörter Schlafrhythmus }\end{array}$ & & $9 \mathrm{Mo}$ \\
\hline SSRIS & $\begin{array}{l}\text { Irritabilität, schrilles Schreien, Unruhe, hoher Mus- } \\
\text { keltonus, vermehrtes Saugbedürfnis, Trinkschwä- } \\
\text { che, Hypoglykämie, Krämpfe, unruhiger Schlaf }\end{array}$ & Stunden - Tage & 1-4 Wochen \\
\hline
\end{tabular}


aber Daten über die Neurotoxizität und die Langzeitfolgen gerade aus Regionen wie den Niederlanden und der amerikanischen Westküste [13]. In einer 1978 begonnenen longitudinalen Studie wurden 84 Schwangere, aus Familien mit mittleren Einkommen stammend, begleitet [21]. Bei den Kindern zeigten sich:

- ab 4. Lebensjahr: niedrigere Werte, sich verbal zu äußern, niedrigere Gedächtnisleistung

- ab 6. Lebensjahr: schlechteres Sprachverständnis, Gedächtnis-, Seh- Wahrnehmungsfunktionen und Leseaufgaben, verminderte anhaltende Aufmerksamkeit

- 9-12 Lebensjahr: keine globalen Intelligenzdefizite oder Defizite auf den verbalen Subskalen der IQTests, sondern Defizite in den Exekutivfunktionen wie Impulskontrolle und visuelles Problemlösen

- 13-16 Lebensjahr: Aufmerksamkeit, Problemlösung, visuelle Wahrnehmung, analytische Fähigkeiten, ausdauernde Aufmerksamkeit vermindert

- 18-22 Lebensjahr: funktionelle Magnetresonanztomographie (fMRI) zeigt Veränderung der neuronalen Aktivität bei Arbeitsgedächtnisaufgaben

Interessant: je höher die Dosis pränatal, desto höher die Dysfunktion auf hyperreaktiven/impulsiven Skalen

\section{Entzugssymptomatik bei Missbrauch} nichtnarkotisierender Medikamente

Ein Vergleich nichtnarkotisierender Medikamente, die psychomotorische Veränderungen analog einer Entzugssymptomatik bei Neugeborenen hervorrufen, findet sich in $>$ Tab. 1.

\section{Langzeitfolgen von Drogenabusus beim Kind}

Neurotoxische Substanzen wie Alkohol und Cannabis sind bezogen auf ihre Langzeitfolgen fürs Kind durch Substanzmissbrauch der Mutter in der Schwangerschaft hervorzuheben. Sie heben sich in ihrem Risiko für Lern-, Verhaltens-, Impulskontrollstörungen deutlich von anderen ab [14][16][17]. Betroffene Jugendliche fallen in der Schule unter strukturierter Förderung möglicherweise wenig auf, finden jedoch schwer in eine Eigenverantwortung und Selbststrukturierung.

Diese „schwierigen“ Kinder leben in Familien, in denen die Eltern ihren Alltag mithilfe von Substanzen regulieren und bewältigen. Hier unterstützend und wenn nötig regulierend einzugreifen, fällt in den Bereich der Jugendämter. Diese Kontakte müssen von Anfang an gebahnt werden. Wenn sie ein fester Bestandteil des Behandlungskonzeptes sind, wird dies gut akzeptiert und es mindert den Beigeschmack der „bösen Kontrollpolizei“.

\section{Therapiestrategien im Wochenbett}

\section{Nichtmedikamentöse Therapie}

Nichtmedikamentöse Therapiestrategien im Wochenbett beziehen an erster Stelle die Mutter ein und helfen ihr, ein besseres Selbstverständnis zu schaffen. Eine große Chance liegt darin, die Mutter sowohl im Umgang mit den Besonderheiten ihres Babys als auch mit ihren eigenen Selbstvorwürfen und Schuldgefühlen anzuleiten.

\section{Sanfte Pflege von Mutter und Kind}

Für eine sanfte Pflege in ruhiger, abgedunkelter Umgebung benötigt man eine ausreichende Personaldecke, um die sinnvolle 1:1-Betreuung umzusetzen. Auch sollte auf eine Kontinuität bei der Pflege geachtet werden. Es gibt nur wenige Situationen, die eine Schwester oder einen Pfleger der Neonatologie so herausfordern wie die Gegebenheit, ein Baby nicht beruhigen zu können. Häufig sind die Pflegenden selbst Eltern und müssen die Mutter des Kindes anleiten und schulen, aus deren Drogenmissbrauch das kindliche Leid resultierte. Dabei gilt es dennoch, ihr mit Respekt und Achtung gegenüberzutreten. Den Schwestern und Pflegern, die diesen inneren Konflikt jeden Tag leben und trotzdem Liebe und Geduld mit Mutter und Kind aufbringen, gehört unser höchster Respekt.

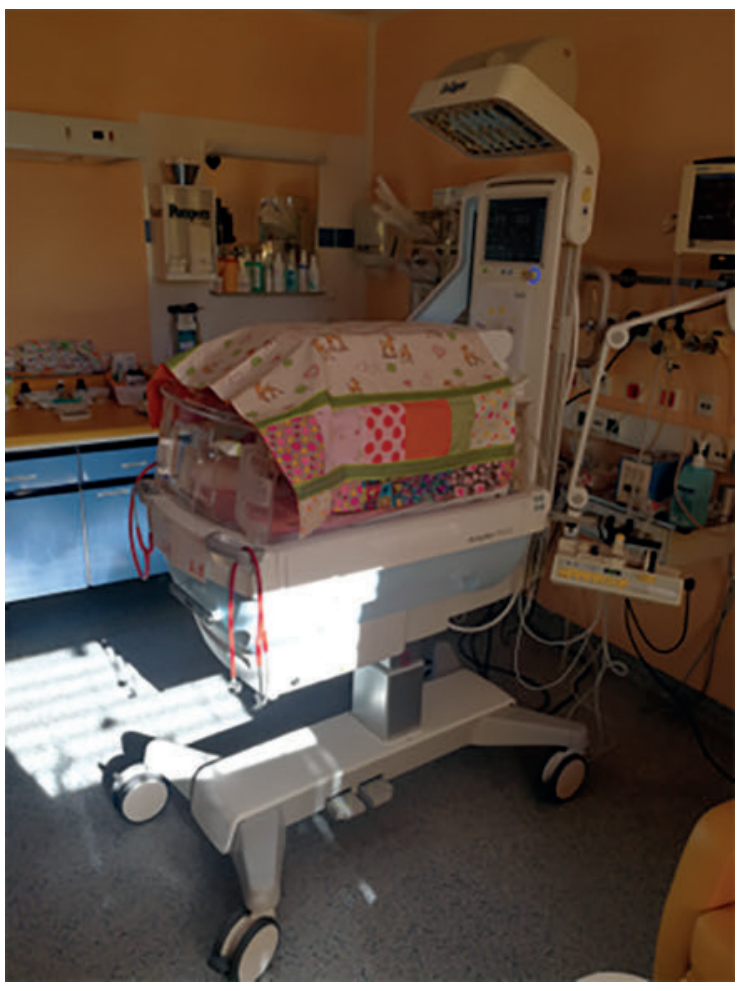

Abb. 1 Optimaler Raum für Neugeborene mit Neonatalem Abstinenzsyndrom: abgedunkelte, ruhige Umgebung mit einem Minimum an Störfaktoren wie Licht oder Geräuschen. (Quelle: Andreas Gerhardt, Harzklinikum Wernigerode, [rerif]) 


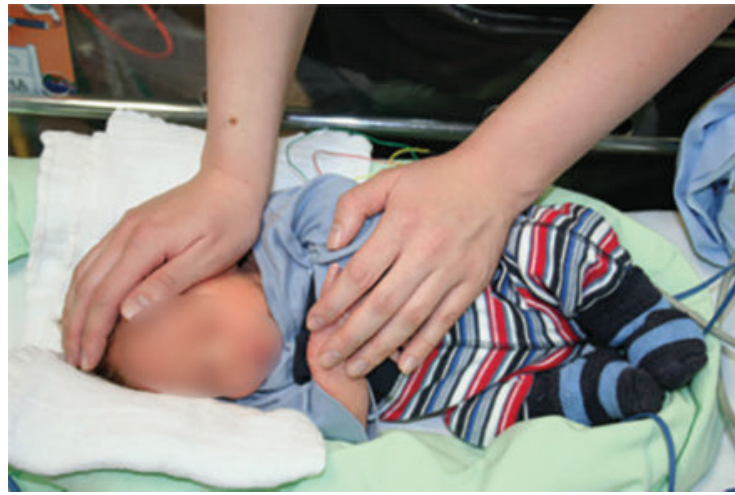

Abb. 2 Beispiele für sanfte Pflege: halten, wiegen, umgrenzen, pucken, facilitated tucking. (Quelle: Andreas Gerhardt, Harzklinikum Wernigerode, [rerif])

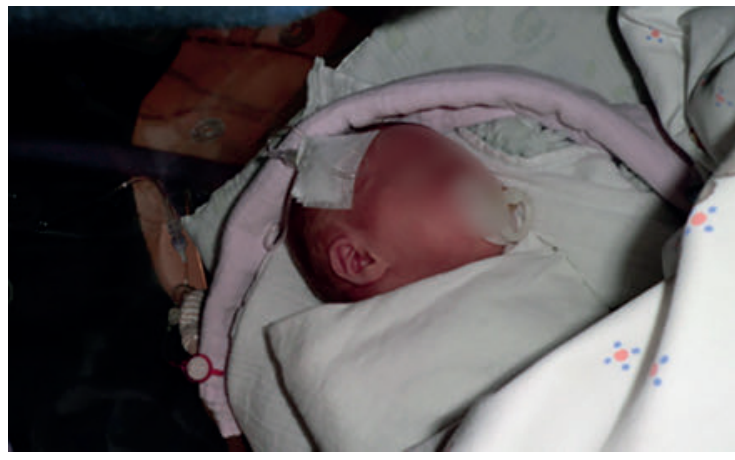

- Abb. 4 Schmerzlinderung mit Glucose $20 \%$ (non-nutritives Saugen). (Quelle: Andreas Gerhardt, Harzklinikum Wernigerode, [rerif])

Gutes Pflegepersonal und ausreichend Zeit ermöglichen es, die medikamentöse Therapie durch diese Strategien deutlich zu begrenzen. Das hat eine Reduktion der Nebenwirkungen, der Nachwirkungen und bei Verkürzung der Therapiedauer auch eine Kostensenkung zur Folge.

\section{Herausforderungen für die Pflege im Wochenbett bei Drogenabusus}

- besondere Interaktion zwischen Mutter und Pflegepersonal

- „Konkurrenz“ um bessere Elternschaft (eigene Vorstellung von Familie)

- gegenseitiges Misstrauen („Mütter glauben, Schwestern wollen Ihnen das Kind wegnehmen ...")

- Mitleid mit dem Kind, Wut auf die Mutter

- Frustration (Regeln vs. Unzuverlässigkeit)

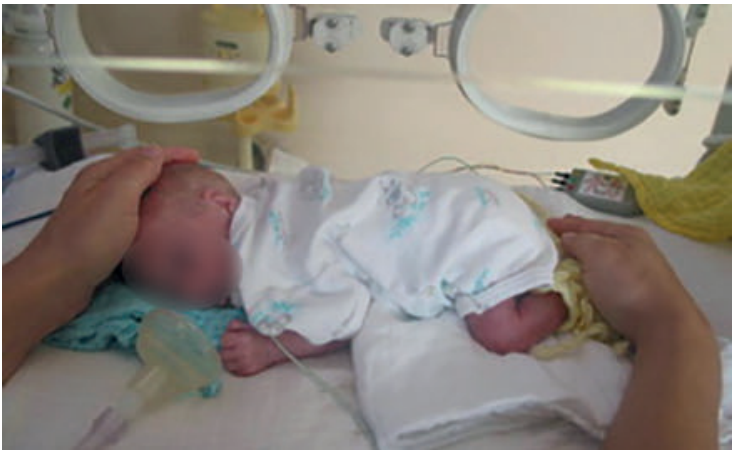

Abb. 3 Ausreichend Zeit und sanfte Pflege können eine Begrenzung der medikamentösen Therapie ermöglichen. (Quelle: Andreas Gerhardt, Harzklinikum Wernigerode, [rerif])

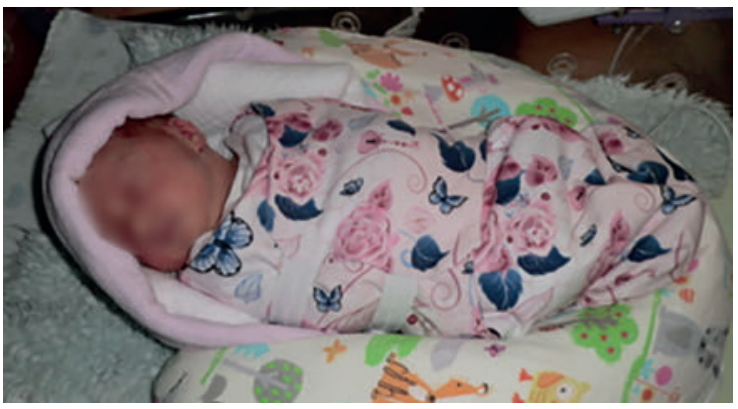

-Abb. 5 Ein geregelter Schlaf-Wach-Rhythmus strukturiert den Tagesablauf. (Quelle: Andreas Gerhardt, Harzklinikum Wernigerode, [rerif])

Respektvoller und wertschätzender Umgang mit Eltern

In der Neonatologie und Wochenbettbetreuung liegt der Fokus auf einem respektvollen und wertschätzenden Umgang mit den Eltern. Obligat ist dabei ein engmaschiges Herzkreislaufmonitoring des Säuglings, um Apnoen und Krampfanfälle zu detektieren. Das Bonding der Mutter an das Kind sollte unmittelbar nach der Geburt erfolgen. Regelmäßiges Kangarooing muss von vielen Müttern erst erlernt werden. Bei fehlender innerer Ruhe der Mutter ist eine enge Kooperation mit den Suchttherapeuten notwendig.

Die Vulnerabilität im Wochenbett, durch die neue Situation, den Schlafmangel, Schmerzen und die hormonelle Umstellung erhöhen das Rückfallrisiko. Tägliche Physiotherapie ergänzt die entwicklungsfördernde Pflege und entlastet die betreuende Schwester. 


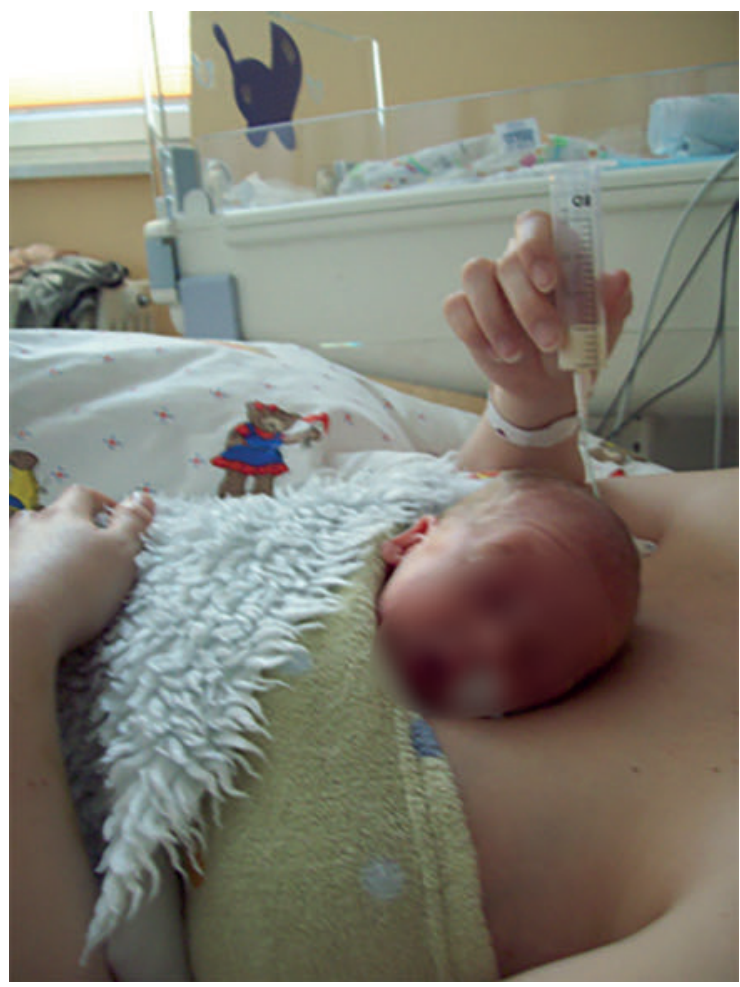

Abb. 6 Fürs Bonding und Kangarooing braucht die Mutter innere Ruhe - ggf. ist eine Kooperation mit den Suchttherapeuten notwendig. (Quelle: Andreas Gerhardt, Harzklinikum Wernigerode, [rerif])

\section{AUS DER PRAXIS \\ Ein Wort zum Stillen bei Müttern mit Suchtproblematik}

Einige Autoren sehen das Stillen bei Suchtkranken als problematisch. Doch nach unseren Erfahrungen möchten viele Mütter stillen und dies fördern wir besonders auch bei Methadon-substituierten Frauen [13]. Da dies nur ohne Beikonsum möglich ist, stimmen sie häufig regelmäßigen Urinkontrollen zu und arbeiten enger mit dem Suchtherapeuten und Drogenberatern zusammen. Die Mutter-Kind-Bindung setzt hier einen Therapiewillen bei der Frau frei und bei Gelingen der Stillbeziehung messen wir diesem auch einen prädiktiven Wert bei.

\section{Medikamentöse Therapie}

Nicht immer korrelieren Substanzdosis oder Dauer des Abusus der Mutter mit der Stärke der Entzugssymptome beim Neugeborenen. Begleitende Umstände, z. B. Infektionen, Geburtskomplikationen oder individuelle Gegebenheiten des Neugeborenen machen manchmal eine medikamentöse Therapie notwendig.

Besonders bei Opiatkonsum, wenn aufgrund von Durchfällen und wundem Po offene Pflege notwendig ist oder

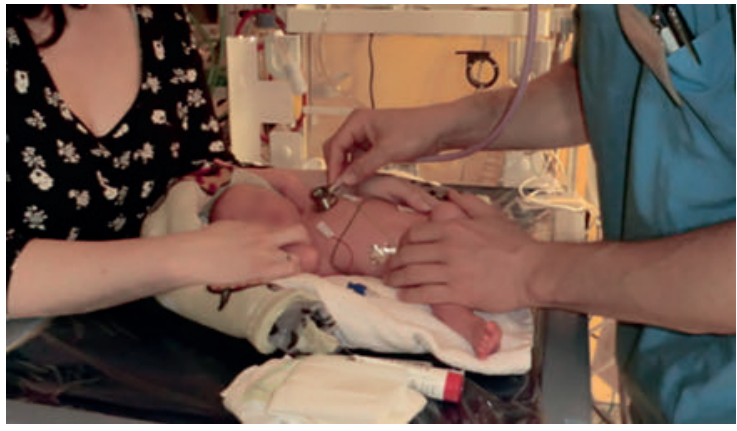

Abb.7 Die Eltern werden wertschätzend und respektvoll behandelt und in die Betreuung ihres Babys einbezogen. (Quelle: Andreas Gerhardt, Harzklinikum Wernigerode, [rerif])

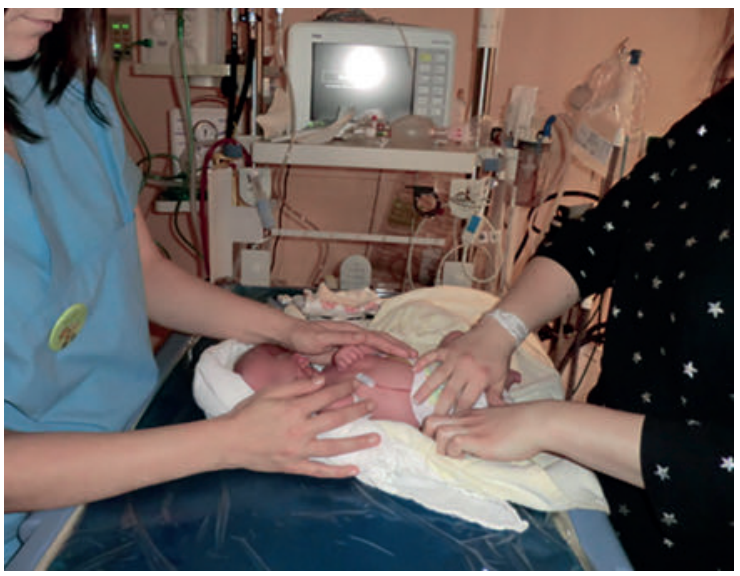

- Abb. 8 Die basale Stimulation durch Physiotherapie hat das Ziel der maximalen Stress- und Schmerzreduktion. (Quelle: Andreas Gerhardt, Harzklinikum Wernigerode, [rerif])

wenn Phototherapie eine Begrenzung durch Pucken des Kindes unmöglich macht, setzen wir orale Morphinverdünnung ein. Diese titrieren wir je nach Schwere von Symptomen, die wir mittels des Finnegan-Scores monitoren ( $\triangleright$ Tab. 2).

Ergänzend applizieren wir Phenobarbital und Clonidin. Unser Eindruck ist aber, dass mehr Substanzen in der Therapie diese sehr verkomplizieren und die Reduktion erschweren.

\section{Die Zeit nach der Klinik}

Rückt die Klinikentlassung näher, beginnen wir, die Mitarbeiter des Jugendamts, der Drogenberatung und die Hebamme für die häusliche Betreuung zu integrieren. Bei gemeinsamen Treffen am „Runden-Tisch“ besprechen wir mit ihnen das weitere Vorgehen, die Hausbesuche und wägen $a b$, ob die Unterbringung in einem Mutter-Kind-Heim sinnvoll ist. Gegebenenfalls wird 
Tab. 2 Finnegan-Score.

\begin{tabular}{|l|l|l|l|l|l|}
\hline Klinisches Kriterium & $\mathbf{1}$ & $\mathbf{2}$ & $\mathbf{3}$ & $\mathbf{4}$ & $\mathbf{5}$ \\
\hline Häufiges Gähnen & ja & - & - & - & - \\
\hline Marmorierte Haut & ja & - & - & - & - \\
\hline Verstopfte Nase & - & ja & - & - & - \\
\hline Niesen & ja & - & - & - & - \\
\hline Atmung & $>60 /$ min & $>60 /$ min, & - & - & - \\
\hline Übermäßiges Saugen & ja & Dyspnoe & - & - & - \\
\hline Trinkschwäche & - & ja & - & - & - \\
\hline Erbrechen & - & Regurgitation & im Schwall & - & - \\
\hline Stühle & - & dünn & wässrig & - \\
\hline
\end{tabular}

schließlich Letztere geplant und organisiert. Oft sind mehrere Treffen notwendig. Ein gleicher, gemeinsamer Wissensstand über die Absprachen schafft Vertrauen und beschleunigt die Umsetzung der Vereinbarungen.

Bei einigen Müttern bestand schon in der Schwangerschaft oder entsteht während der Entzugsphase ihres Babys der Wunsch nach einer Rehabilitation. Für uns ist dahingehend die enge Zusammenarbeit mit einer Suchtklinik ein großer Bonus. Manche Frauen beginnen dort während der Schwangerschaft schon ihre Therapie. Geburtshilflich werden sie in unserem Perinatalzentrum begleitet. Um die Geburt zieht die Mutter auf die Wochenstation und die Suchttherapeuten begleiten sie bei uns kontinuierlich, bis das Neugeborene nach überstandenem Entzug oder nach Frühgeburtlichkeit von unserer Neonatologie entlassen werden kann. Dann erfolgt die Fortsetzung der mütterlichen Suchttherapie in der Rehaklinik und unser Hebammenteam begleitet sie. In dieser Kontinuität liegt für die Mutter die Möglichkeit, schneller Vertrauen zu fassen. Zudem sichert sie den betreuenden Disziplinen einen gleichen Informationsstand.

\section{Wünsche für die Zukunft}

Auch in unserem Perinatalzentrum liegen Wünsche und Ideen oft fern von gelebter Realität. Was bereits in Einzelfällen erfolgreich praktiziert wurde, ist jedoch weit entfernt von einer eingeübten Routine. Wir wünschen uns trotz Personalknappheit und geringen zeitlichen Ressourcen:

- Engmaschigere Betreuung im Zeitraum der Geburt (3 Monate vor und nach der Geburt)

- Optimierung der Koordination für den „Runden Tisch“ aus Substitutionsärzten, Hebammen, Jugendamt, Sozialdienst
- Psychologische Unterstützung und Deeskalationstraining für unser Pflege-Team

- Mehr Rückmeldung der ambulanten Kinderärzte über den weiteren Verlauf der kleinen Patienten

\section{Autorinnen / Autoren}

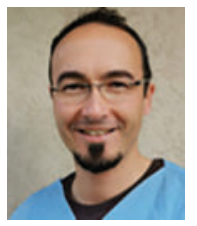

Dr. med. Andreas J. Gerhardt ist Oberarzt Kinder- und Jugendmedizin, Schwerpunkt Neonatologie, am Harzklinikum Wernigerode.

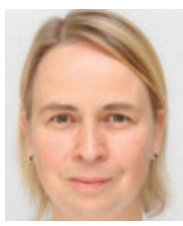

Dr. med. Carmen Aschka ist Oberärztin Gynäkologie und Geburtshilfe, Schwerpunkt Spezielle Geburtshilfe und Perinatalmedizin, am Harzklinikum Wernigerode.

\section{Korrespondenzadresse}

Harzklinikum Dorothea Christiane Erxleben Wernigerode Ilsenburger Straße 15

38855 Wernigerode

E-Mail: carmen.aschka@harzklinikum.com

E-Mail: andreas.gerhardt@harzklinikum.com

\section{Literatur}

[1] AWMF RegNr. 022-025, S3-Leitlinie Diagnose der Fetalen Alkoholspektrumstörungen; 2016

[2] AWMF RegNr. 022-025, S3-Leitlinie Diagnose der Fetalen Alkoholspektrumstörungen; 2016

[3] AWMF RegNr. 015-065, Leitlinie Vorgehen bei Terminüberschreitung und Übertragung; 2014 
[4] AWMF RegNr. 038-024, S3-Leitlinie Methamphetaminbezogene Störungen; 2016

[5] Bergmann RL, Richter R, Milto C et al. Epidemiologie des Alkoholkonsums in der Schwangerschaft. In: Bergmann RL, Spohr $\mathrm{H}$-L, Dudenhausen JW (Hrsg.). Alkohol in der Schwangerschaft - Häufigkeit und Folgen. München: Urban und Vogel; 19-32

[6] Seitz NN, Lochbühler K, Atzendorf J et al. Trends des Substanzkonsums und substanzbezogener Störungen. Auswertung des Epidemiologischen Suchtsurveys von 1995 bis 2018. Deutsches Ärzteblatt International 2019; 116 (35-36), 585591. Im Internet: https: / /www. esa-survey.de/publikationen/ fachliteratur/fachliteratur-detailansicht/lm/trends-des-sub stanzkonsums-und-substanzbezogener-stoerungen-auswer tung-des-epidemiologischen-suchtsu.html (abgerufen am 14.6.2020)

[7] Drogen- und Suchtbericht 2018 - Drogenbeauftrage (BMG-D). Im Internet: https://www.bundesregierung.de/breg-de/such e/drogen-und-suchtbericht-2018-1545480 (abgerufen am 14. 6.2020)

[8] Drogen- und Suchtbericht 2019 - Drogenbeauftrage (BMG-D). Im Internet: https: / /www.drogenbeauftragte.de/fileadmin/da teien-dba/Drogenbeauftragte/4_Presse/1_Pressemitteilungen/ 2019/2019_IV.Q/DSB_2019_mj_barr.pdf (abgerufen am 25. 6.2020)

[9] Rauschgiftkriminalität Bundeslagebild 2016/2017- Bundeskriminalamt. Im Internet: https://www.bka.de/SharedDocs/ Downloads/DE/Publikationen/JahresberichteUndLagebilder/R auschgiftkriminalitaet/2016RauschgiftBundeslagebildZ.html;j sessionid $=$ F58CD702DFC24317C3BF2756F5D5E629. Iiv e2302?nn= 27972 (abgerufen am 14.6.2020)

[10] Hoch E, Friemel CM, Schneider M. Cannabis: Potential und Risiken. Eine wissenschaftliche Analyse. Im Internet: https: / /www.drogenbeauftragte.de/fileadmin/dateien-dba/ Drogenbeauftragte/2_Themen/2_Suchtstoffe_und_Abhaen gigkeiten/6_Cannabis/Downloads/BMG_CaPris_A5_Info_web. pdf (abgerufen am 14.6.2020)

[11] Deutsches Ärzteblatt 23.6.2016. Schwangerschaft: Cannabis stört Hirnentwicklung anders als Rauchen. Im Internet: https: / /www.aerzteblatt.de/nachrichten/69252/Schwanger schaft-Cannabis-stoert-Hirnentwicklung-anders-als-Rauchen (abgerufen am 14. 6.2020)
[12] Deutsches Ärzteblatt 24.6.2015. US-Gynäkologen warnen vor Marihuana in Schwangerschaft und Stillzeit. Im Internet: https: / /www.aerzteblatt.de/nachrichten/63246/US-Gynae kologen-warnen-vor-Marihuana-in-Schwangerschaft-und-Stil Izeit (abgerufen am 14. 6.2020)

[13] Ryan SA, Ammerman SD, O'Connor ME. Marijuana Use During Pregnancy and Breastfeeding: Implications for Neonatal and Childhood Outcomes Pediatrics 2018; 142 (3) e20181889; DOI: $10.1542 /$ peds.2018-1889

[14] Hudak ML, Tan RC. The Committee on Drugs and the CommitteeFetus and Newborn. Neonatal Drug Withdrawal. Pediatrics 2012; 129; e540

[15] Hudak ML, Tan RC, The Committee on Drugs and the Committee on Fetus and Newborn. Neonatal Drug Withdrawal. Pediatrics. 2012;129; 540. Pediatrics 2014; 133 (5) 937-938; DOI: https://doi.org/10.1542/peds.2014-0557

[16] Hudak ML, Tan RC. The committee on drugs, the committee on fetus and newborn. Pediatrics 2012, 129 (02) e540-e560; DOI: 10.1542/peds.2011-3212

[17] Hwang SS, Barfield WD, Smith RA et al. Discharge timing, outpatient and home care of late-preterm and earlyterm Pediatrics 2013; 132 (01): 101-108

[18] Lynch S, Sherman L, Snyder Susan et al. Trends in infants reported to child welfare with neonatal abstinence syndrome (NAS). Children and Youth Services Review 2018. 86: 135141. DOI: $10.1016 /$ j.childyouth.2018.01.035

[19] Drogen- und Suchtbericht 2019 - Drogenbeauftrage (BMG-D)

[20] Landgraf MN. Fetale Alkoholspektrumstörung - Diagnose und frühe Förderung. Die Hebamme 2017; 30: 336-345

[21] Fried PA. The Ottawa Prenatal Prospective Study (OPPS): methodological issues and findings - it's easy to throw the baby out with the bath water. Life Sci 1995; 56 (23-24): 2159-68

Bibliografie

DOI https://doi.org/10.1055/a-1213-8106

Die Hebamme 2020; 33: 30-39

(c) Georg Thieme Verlag KG Stuttgart · New York ISSN 0932-8122 